\title{
Autonomic Alterations in Cocaine-Exposed Neonates Following Orthostatic Stress
}

\author{
VIJAY JOHN, HONGYING DAI, AJAY TALATI, RICHARD J. CHARNIGO, MICHAEL NEUMAN, AND HENRIETTA S. BADA
}

Joint Biomedical Engineering Program [V.J.], Department of Pediatrics [A.T.], University of Tennessee, Memphis, TN, 38163; Department of Mathematics [H.D.], Columbus State University, Columbus, GA, 31907; Department of Statistics [R.J.C.], and Department of Pediatrics [H.S.B.], University of Kentucky, Lexington, KY, 40536; Department of Biomedical Engineering [M.N.], MI Technological University, Houghton, MI, 49931

\begin{abstract}
We investigated the effects of prenatal cocaine exposure (PCE) on heart rate (HR) and heart rate variability (HRV) in the presence of orthostatic stress among near- and full-term neonates. PCE infants $(n=21)$ and controls $(n=23)$ were enrolled within $120 \mathrm{~h}$ of birth. ECG was recorded for an hour during quiet sleep, 30 min in supine position and then $30 \mathrm{~min}$ in an inclined position. Linear mixed models were used to analyze HR and HRV in the time domain and wavelet and power spectrum analyses in the frequency domain. PCE infants had tachycardia both before $(p=0.091)$ and after tilting $(p=0.015)$, but with a clear interaction between PCE and orthostatic stress $(p=0.049)$. Compared with controls, PCE infants had a delayed and prolonged reaction to orthostatic stress. There was also a pronounced interaction with regard to $\log$-transformed SDDRR, a measure of HRV ( $p=0.049)$. Controls experienced an instantaneous increase in $\log$ (SDDRR) followed by a prompt return to normal levels, while PCE infants had a gradual increase that did not dissipate quickly. Frequency-domain analyses also distinguished between the cocaine-exposed infants and the controls. Results suggest that the effects of PCE on the development of sympathetic and parasympathetic systems could lead to altered cardiovascular function. (Pediatr Res 61: 251-256, 2007)
\end{abstract}

$\mathrm{H}$ eart rate (HR) and heart rate variability (HRV) are increasingly used to detect a variety of pathophysiologic alterations in the autonomic nervous system (ANS) regulation of cardiac control mechanisms (1). Infants who succumb to sudden infant death syndrome have higher HR and reduced HRV compared with other infants; one speculation is that autonomic disturbances may lead to cardiac instability or may indicate ANS alterations with the potential to affect other vital functions (2). Moreover, the circadian range of HR and certain quantities related to blood pressure (BP) are predictors of neonatal cardiovascular events (3). Hence, power spectrum analysis (PSA) of HRV has been used to assess fetal viability and maturity of the ANS $(4,5)$.

Cocaine use by pregnant women has continued to be a prevalent public health problem. Prenatal cocaine exposure (PCE) may lead to abruptio placentae, premature delivery,

Received June 1, 2006; accepted September 16, 2006.

Correspondence: Henrietta S. Bada, M.D., University of Kentucky, KY Children's Hospital, 800 Rose Street Room MS 477, Lexington, KY 40536 USA; e-mail: hbada2@uky.edu

The study was funded in part by a grant from the Children's Miracle Network, KY Children's Hospital.

DOI: $10.1203 / 01 . p d r .0000252436 .62151 .67$ intrauterine growth restriction, congenital malformations, and fetal death (6-8). However, controversy exists regarding the influences of PCE on the ANS control mechanism and on neonatal cardiovascular function. Some studies (9-11), have found elevated HR and reduced HRV in infants with PCE. On the other hand, Regalado et al. (12) found reduced HR and increased HRV following PCE, while another study focusing on the nonlinear dynamics of HRV (13) found no significant differences between PCE infants and controls in spectral power distribution, approximate entropy, correlation dimension, or nonlinear predictability. Spectral analyses in particular have not yielded a consensus about the nature of PCEinduced autonomic alterations. Higher HRV was attributed to increased spectral power across all frequency bands in quiet sleep and to increased spectral power across low-frequency $(0.03-0.1 \mathrm{~Hz})$ and mid-frequency $(0.1-0.2 \mathrm{~Hz})$ bands in active sleep (14). In contrast, Oriol et al. (9) found that spectral power during ten-minute periods of quiescent sleep was significantly smaller in infants with PCE. The apparent conflict among study results may be due partly to the differing demographic characteristics of the populations from which the samples were taken. Perhaps more importantly, numerous measures have been proposed to quantify HR and HRV; analyses based on different measures can lead to different conclusions.

Such controversy motivates the present study, in which we seek greater understanding of autonomic regulation and cardiac control mechanisms in infants with PCE compared with nonexposed infants. What distinguishes this study from those that have preceded it is that the infants were subjected to an orthostatic stress: specifically, a $25^{\circ}$ head-up tilt took place during quiet sleep. Although the present study is not the first to involve orthostatic stress, we believe it is the first to investigate the effects of PCE on neonatal HR dynamics in response to orthostatic stress.

Regarding methodology for HR and HRV analysis, there has been a dichotomy in the literature; some analyses are

\footnotetext{
Abbreviations: ANS, autonomic nervous system; BP, blood pressure; corSDRR, corrected SDRR; HR, heart rate; HRV, heart rate variability; LMM, linear mixed model; PCE, prenatal cocaine exposure; PSA, power spectrum analysis; SDRR, standard deviation of the R-R intervals; SDDRR, standard deviation of differences between successive R-R intervals
} 
based on time-domain measures, while others rely on frequency-domain measures. PSA and nonlinear analysis are commonly used since they define a full spectrum for R-wave inter-beat dynamics and can provide insights about the sources of autonomic alterations. However, frequency-domain approaches have the following limitations: 1) Information about the time sequence of HR dynamics can be lost. Without this information, it is difficult to assess how PCE affects the reaction to a specific intervention such as orthostatic stress; 2) Some frequency-domain approaches assume a certain kind of stability in the time series data; such an assumption may not be tenable when measurements take place both before and after an intervention $(15,16)$; and 3 ) There is some disagreement about how to define high frequency and low frequency. Moreover, different summary statistics have been used to measure power over a frequency band, and final conclusions may be sensitive to the manner in which power is measured. 4) Frequency-domain quantities can be influenced by artifacts, requiring additional screening or noise reduction by the operator. Time-domain quantities are comparatively operatorindependent (10).

The above points are not meant to impugn frequencydomain analyses but rather to explain why time-domain analyses are central to the present study, which concerns HR dynamics before and after an intervention. Temporal trajectories of HR and HRV are of primary importance as we seek to characterize the magnitudes, profiles, and durations of autonomic alterations.

\section{SUBJECTS AND METHODS}

Twenty-one infants with PCE were enrolled into the study. PCE was determined by maternal self-report or neonatal urine toxicology. Nine mothers who used cocaine also used other substances ( 3 - marijuana, 2 - marijuana + tobacco, 1 - marijuana and alcohol, and 3 - tobacco and alcohol). In the control group $(n=23)$, infants tested negative for cocaine; their mothers had no history of drug use, and were selected from the same population of African-American women as the mothers of PCE infants. Babies were recruited from the well baby nursery and neonatal intensive care unit (NICU) at the Regional Medical Center in Memphis, TN. All babies were examined within $120 \mathrm{~h}$ of birth. Written informed consent was obtained from each mother. Both Institutional Review Boards of the University of Tennessee and the University of Memphis approved the protocol.

Protocol and recording procedure. Each infant had electrocardiogram (ECG) monitoring. A tilt table was placed under the infant's mattress, the infant was strapped to the table, and the table could be manually tilted to an angle of $25^{\circ}$. Two 30-min segments of the ECG were recorded, the first with the infant supine, and the second with the infant inclined in a $25^{\circ}$ head-up position. ECG recordings were made with the infants in a state of quiet sleep (eyes closed, regular respiration, and no gross body movement). Recordings in the NICU were obtained with the Hewlett Packard M1046-9021C neonatal monitoring system (Palo Alto, CA), and in the well baby nursery with the NIMS Respitrace PT monitoring system (North Bay Village, FL), originally designed for the Collaborative Home Infant Monitoring Evaluation (CHIME) study (17). The R-wave peaks from each cardiac cycle in the Hewlett Packard recordings were determined using a peak detection algorithm (18); the R-R intervals had a resolution of $2 \mathrm{~ms}$. The CHIME software used with the NIMS system generated R-R intervals with $1 \mathrm{~ms}$ resolution.

ECG artifact correction. The Hewlett Packard recordings contained noise of both biologic and environmental origins. Noise was identified and removed through careful screening of the ECG wave form with the R-wave peak detection algorithm. After noise reduction, the ECG wave form was again input into the R-wave peak detection algorithm. The NIMS system recordings were artifact-free.

$\boldsymbol{H R}$ and $\boldsymbol{H R V}$ analysis. Both time-domain and frequency-domain quantities were analyzed. Time-domain data included HR in beats per minute (bpm), $\mathrm{SD}$ of the R-R intervals (SDRR), and SD of differences between successive
R-R intervals (SDDRR). SDRR is a measure of HRV that is influenced by the length and dispersion of R-R intervals. The length of R-R intervals is related to the parasympathetic control of HR, while the dispersion of R-R intervals reflects both vagal and sympathetic modulations (19). Since larger SDRR may be primarily due to longer R-R intervals rather than increased dispersion, we also considered a new quantity called the corrected SDRR (corSDRR); i.e. corrected for HR:

$$
\text { corrected SDRR }=\frac{\mathrm{SDRR}}{\text { mean of } \mathrm{RR}} \approx \mathrm{SDRR} \times \text { mean of } \mathrm{HR}
$$

CorSDRR reflects dispersion of R-R intervals but not length. SDRR, SDDRR, and corSDRR were calculated using a 30-s window moved in 5-s increments; the SDRR, SDDRR, and corSDRR for each window were then assigned to the midpoint time of the window. So, for example, the SDRR at $15 \mathrm{~s}$ before tilt was based on data from $30 \mathrm{~s}$ before tilt to the time of tilting; the next SDRR was based on data from $25 \mathrm{~s}$ before tilt to $5 \mathrm{~s}$ after tilt.

Frequency-domain analyses for this study included both PSA and wavelet analysis; the former is based on a Fourier transform and assumes that data are wide-sense stationary $(15,16)$. In PSA, the low frequency (LF) region $(0.02-$ $0.2 \mathrm{~Hz})$ is viewed as a marker of both sympathetic and parasympathetic activation (20), while the high frequency (HF) region $(0.2-2 \mathrm{~Hz})$ is treated as a marker of parasympathetic activation (21). Low-frequency power $\left(\mathrm{P}_{\mathrm{L}}\right)$ is the integral of the power function over the LF region, high-frequency power $\left(\mathrm{P}_{\mathrm{H}}\right)$ is the integral of the power function over the HF region, and total power $\left(\mathrm{P}_{\mathrm{T}}\right)$ is the sum of the two. The ratios $\mathrm{P}_{\mathrm{L}} / \mathrm{P}_{\mathrm{T}}, \mathrm{P}_{\mathrm{H}} / \mathrm{P}_{\mathrm{T}}$, and $\mathrm{P}_{\mathrm{L}} / \mathrm{P}_{\mathrm{H}}$ reflect the balance between sympathetic and parasympathetic activation.

Wavelet analysis tracks the temporal evolution of frequencies contained in time-series data (22). Specifically, a wavelet transform converts time-domain data into a collection of wavelet coefficients, some of which represent HF content and some of which represent LF content. A wavelet transform is like a Fourier transform, but there is an important difference: wavelet functions are localized in time, so that wavelet coefficients possess a temporal structure. Hence, a wavelet power function can be defined with time as the input. For this study, the wavelet power function was defined as the maximal wavelet coefficient among a collection of coefficients associated with a certain time. The wavelet power function was then decomposed into HF and LF wavelet power functions according to whether the maximal coefficient represented HF content or LF content. The integrals of the HF and LF wavelet power functions over the time periods when the infants were horizontal or head-up were defined as the HF and LF wavelet power for those time periods.

Statistical methods. Data were analyzed using SAS (SAS Institute, Cary, NC), SIGMASTAT (Systat Software, Point Richmond, CA), and MATLAB (Mathworks, Natick, MA). Statistical significance was defined by a $p<0.05$. For comparison of demographics and baseline characteristics, we used $t$-tests for continuous attributes, Fisher's exact test for dichotomous attributes, and Kolmogorov-Smirnov tests for discrete attributions.

For the time-domain analyses, linear mixed models (LMM) were fit to HR and log-transformed HRV data, as the distributions of untransformed SDRR, SDDRR, and corSDRR were right-skewed. Time was treated as a categorical variable to provide flexibility in modeling the temporal dynamics of HR and HRV. The LMM included both fixed effects and random effects. The random effects were included to accommodate intrasubject correlations. Ignoring these correlations could lead to inaccurate conclusions about the fixed effects; condensing the data to circumvent these correlations would discard information about the temporal trajectories of HR and HRV. We chose variancecovariance structures for the LMM by comparing the values of Akaike Information Criteria and Bayesian Information Criteria obtained with different variance-covariance structures, but variance and covariance parameters whose estimates were less than twice their standard errors were eliminated. Post hoc tests were conducted to investigate the magnitudes and durations of changes associated with the orthostatic stress; global tests were performed before the post hoc tests to ensure control of Type I error probabilities.

For the wavelet analysis, two more LMM were fit: one had as the response LF wavelet power, the other had as the response HF wavelet power, and both models included group, position, and their interaction as predictors. For the PSA, $t$-tests (or, in cases of strong departures from normality, nonparametric alternatives) were used to compare $\mathrm{P}_{\mathrm{L}} / \mathrm{P}_{\mathrm{T}}, \mathrm{P}_{\mathrm{H}} /$ $\mathrm{P}_{\mathrm{T}}$, and $\mathrm{P}_{\mathrm{L}} / \mathrm{P}_{\mathrm{H}}$ at the two positions within each group and between the two groups at each position.

\section{RESULTS}

Characteristics of enrolled subjects. Demographics and baseline characteristics for the two groups are summarized in Table 1. Mothers who used cocaine were older than mothers 
Table 1. Demographics and baseline characteristics for the PCE and control groups

\begin{tabular}{lcc}
\hline \multicolumn{1}{c}{ Characteristics } & Cocaine $(n=21)$ & Control $(n=23)$ \\
\hline Maternal age (years) $\dagger$ & $29.6 \pm 1.7$ & $23.6 \pm 1.1^{*}$ \\
Infants' age (hours) $\dagger$ & $41 \pm 5$ & $39 \pm 6$ \\
No. admitted to NICU/well & $4 / 17$ & $8 / 15$ \\
$\quad$ baby nursery & $13 / 8$ & $10 / 13$ \\
No. male/female & $2924 \pm 117$ & $3113 \pm 101$ \\
Birth weight (grams) $\dagger$ & $47.9 \pm 0.7$ & $49.4 \pm 0.7$ \\
Birth length (cm) $\dagger$ & $32.6 \pm 0.4$ & $33.6 \pm 0.3$ \\
Head circumference (cm) $\dagger$ & 8 & 8 \\
1-min Apgar score, median & 9 & 9 \\
5-min Apgar score, median & &
\end{tabular}

$\dagger$ Values expressed as mean \pm SEM.

$* p=0.004$; all other comparisons: $p \geq 0.05$.

of control infants $(p=0.004)$. No differences were noted between PCE and control groups as to the infants' age at enrollment, gender, birth measurements, Apgar scores, or percentages admitted to the NICU.

Changes in HR. Since any effects of the orthostatic stress would be predominantly instantaneous, the HR from $15 \mathrm{~s}$ before tilt to $15 \mathrm{~s}$ after tilt were analyzed and summarized in Fig. 1. The LMM results are presented in Table 2. There was a significant main effect for group $(p=0.024)$ but not for time ( $p=0.104)$. In the $15 \mathrm{~s}$ preceding tilt, HR was marginally higher among PCE infants $(140.53 \pm 3.11 \mathrm{bpm})$ than among controls $(133.16 \pm 2.97 \mathrm{bpm}, p=0.091)$. During the $15 \mathrm{~s}$ following tilt, mean HR was significantly higher in exposed infants $(147.01 \pm 3.11 \mathrm{bpm})$ than in controls $(136.27 \pm 2.97$ $\mathrm{bpm}, p=0.015)$. There was a significant interaction between group and time $(p=0.049)$, prompting the post hoc tests with results shown in Table 2. The PCE infants had a significant sustained increase in HR during the $15 \mathrm{~s}$ after tilt compared with the $15 \mathrm{~s}$ pre-tilt (increase of $6.48 \pm 2.85 \mathrm{bpm}, p=$ $0.024)$. The controls had also an increase at the time of tilt but transient, only for a duration of $2 \mathrm{~s}$ (increase $=4.69 \pm 2.28$ bpm, $p=0.040$ ). Moreover, the controls responded to the tilt

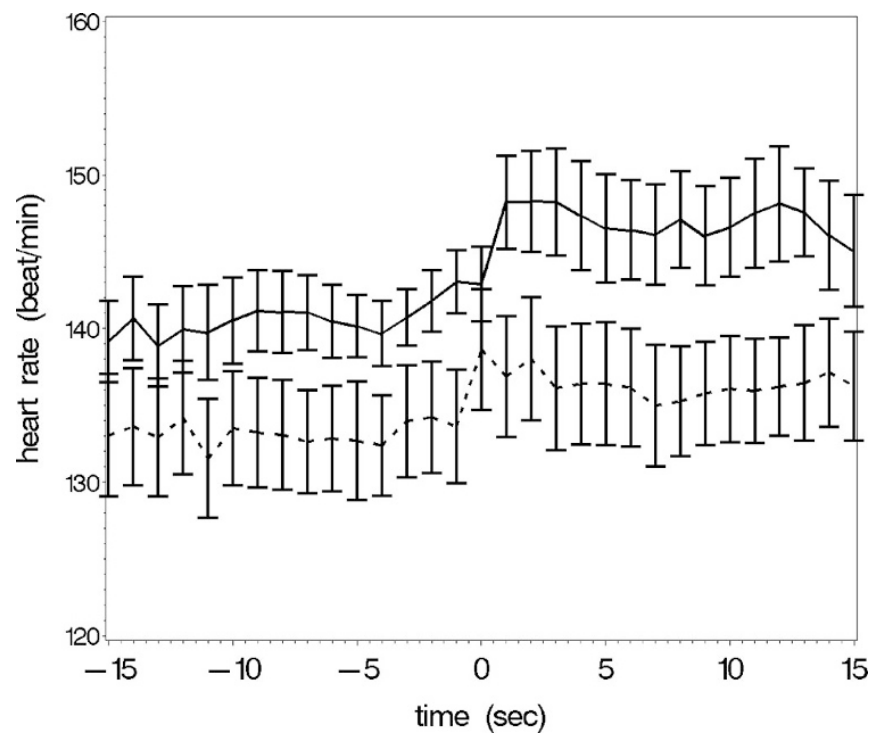

Figure 1. Group means and standard errors for HR within $15 \mathrm{~s}$ of tilting (* time 0 is the tilt time); - Cocaine $(n=21)$, -- Control $(n=23)$.
Table 2. Post hoc comparisons for HR within 15 seconds of tilting

\begin{tabular}{lc}
\hline \multicolumn{1}{c}{ Comparison of means } & Estimate \pm SE \\
\hline PCE vs. Control, 15-s segment pre-tilt & $7.3726 \pm 4.3010 \S$ \\
PCE vs. Control, 15-s segment post-tilt & $10.7473 \pm 4.3010^{*}$ \\
PCE: 15 -s segments pre- \& post-tilt & $6.4802 \pm 2.8528^{*}$ \\
PCE: during 15 -s pre-tilt vs. 2 s post-tilt & $5.9337 \pm 2.3818^{*}$ \\
Control: 15 -s segments pre- vs. post-tilt & $3.1055 \pm 2.7259$ \\
Control: 15 -s pre- vs. during tilt till $2 \mathrm{~s}$ & $4.6891 \pm 2.2759^{*}$ \\
$\quad$ post-tilt & \\
PCE shift, 1 -s pre-tilt to time of tilt, vs. & $-5.1399 \pm 1.5582^{* *}$ \\
$\quad$ Control shift, 1 -s pre-tilt to time of tilt & \\
PCE shift, time of tilt to 1-s post-tilt, vs. & $7.0886 \pm 1.5582 \dagger$ \\
$\quad$ Control shift, time of tilt to 1-s post-tilt & \\
\hline$* 0.01 \leq p<0.05$. & \\
$* * 0.0001 \leq p<0.01$. & \\
$\dagger p<0.0001$. & \\
$\S 0.05 \leq p<0.1$.
\end{tabular}

instantaneously, while PCE infants had a delayed response. The increase in HR from $1 \mathrm{~s}$ before tilt to the time of tilt was higher for controls (difference $=5.14 \pm 1.56 \mathrm{bpm}, p=$ 0.001 ), while the increase from tilt to $1 \mathrm{~s}$ after tilt was higher for cocaine-exposed infants (difference $=7.09 \pm 1.56 \mathrm{bpm}$, $p<0.001)$.

$\log$ (SDDRR). The LMM for log (SDDRR) was fit using the data from $30 \mathrm{~s}$ before tilt to $30 \mathrm{~s}$ after tilting. This accords with the time interval used in the model for HR since information from $15 \mathrm{~s}$ before tilt was required to calculate the value of $\log$ (SDDRR) at $30 \mathrm{~s}$ before tilting. Data are summarized in Fig. 2, and the LMM results are presented in Table 3. There was no significant main effect for group, but there was a significant main effect for time $(p<0.001)$, with interaction between group and time $(p=0.049)$.

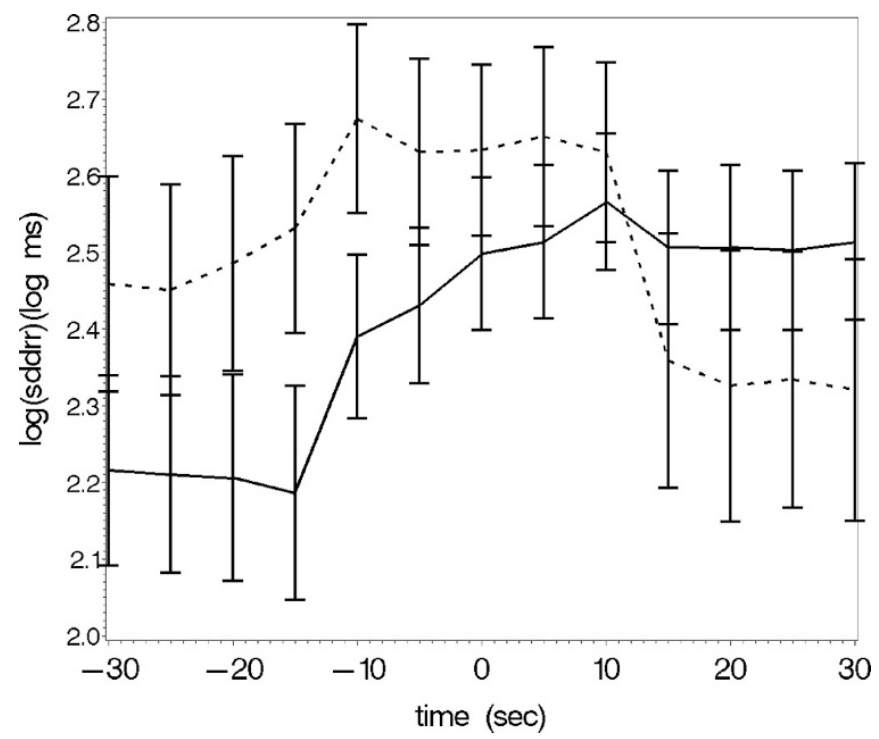

Figure 2. Group means and standard errors for $\log$ (SDDRR) within $30 \mathrm{~s}$ of tilting. The SDRR, SDDRR, and corrected SDRR were calculated using a 30-s window moved in 5-s increments; the SDRR, SDDRR, and corrected SDRR for each window were then assigned to the midpoint time of the window. We defined a pre-tilting segment (times: $-30,-25,-20 \mathrm{~s}$ ), an intra-tilting segment (times: $-15,-10,-5,0,5,10,15 \mathrm{~s}$ ), and a post-tilting segment (times: 20, 25, $30 \mathrm{~s})$. - Cocaine $(n=21)$; --- Control $(n=23)$. 
Table 3. Post hoc comparisons for log (SDDRR) within 30 seconds of tilting

\begin{tabular}{lc}
\hline \multicolumn{1}{c}{ Comparison of means } & $\log ($ SDDRR $) \pm \mathrm{SE}$ \\
\hline PCE: intra-tilt vs. pre- and post-tilt & $-0.0826 \pm 0.0482 \S$ \\
PCE: pre-tilt vs. intra- and post-tilt & $0.2509 \pm 0.0853^{* *}$ \\
PCE: pre-tilt vs. intra-tilt & $0.2312 \pm 0.0772^{* *}$ \\
PCE: intra-tilt vs. post-tilt & $0.06593 \pm 0.0772$ \\
PCE: pre-tilt vs. post-tilt & $0.2971 \pm 0.1206^{*}$ \\
Control: intra-tilt vs. pre- and post-tilt & $-0.1912 \pm 0.0461 \dagger$ \\
Control: pre-tilt vs. intra- and post-tilt & $0.0441 \pm 0.0815$ \\
Control: pre-tilt vs. intra-tilt & $0.1221 \pm 0.0738 \S$ \\
Control: intra-tilt vs. post-tilt & $-0.2603 \pm 0.0738^{* *}$ \\
Control: pre-tilt vs. post-tilt & $-0.1381 \pm 0.1152$ \\
\hline$* 0.01 \leq p<0.05$. & \\
$* * 0.0001 \leq p<0.01$. & \\
$\dagger p<0.0001$. & \\
$\S 0.05 \leq p<0.1$.
\end{tabular}

We therefore partitioned the interval from $30 \mathrm{~s}$ before tilt to $30 \mathrm{~s}$ after tilt into three segments: a pre-tilt segment (times: $-30,-25,-20 \mathrm{~s}$ ), an intra-tilt segment (times: $-15,-10$, $-5,0,5,10,15 \mathrm{~s}$ ), and a post-tilt segment (times: 20, 25, $30 \mathrm{~s})$. Thus, $\log$ (SDDRR) in the pre-tilt segment would not be affected by orthostatic stress, log (SDDRR) in the intra-tilt segment would be influenced by whatever instantaneous effects the tilting had, and log (SDDRR) in the post-tilt segment would be influenced by whatever sustained effects the tilting had.

For control infants, there was a marginal increase in $\log$ (SDDRR) from the pre-tilt segment to the intra-tilt segment (increase $=0.12 \pm 0.07 \log \mathrm{ms}, p=0.099$ ) and then a pronounced decrease from the intra-tilt segment to the post-tilt segment (decrease $=0.26 \pm 0.07 \log \mathrm{ms}, p=0.001)$. Hence, $\log$ (SDDRR) was noticeably greater inside the intra-tilt segment compared with either baseline or post-tilt segments (difference $=0.19 \pm 0.05 \log \mathrm{ms}, p<0.001) . \log ($ SDDRR) was not appreciably different between the pre- and post-tilt segments $(p=0.232)$. The PCE infants reacted quite differently. There was an increase in $\log$ (SDDRR) from the pre-tilt segment to the intra-tilt segment (increase $=0.23 \pm 0.08 \mathrm{log}$ $\mathrm{ms}, p=0.003)$, with the elevated levels of $\log$ (SDDRR) persisting from the intra-tilt segment into the post-tilt segment. Consequently, $\log$ (SDDRR) during the intra-tilt and post-tilt segments was markedly higher than during the pre-tilt segment (difference $=0.25 \pm 0.09 \log \mathrm{ms}, p=0.004$ ). Finally, $\log$ (SDDRR) peaked at different times for the two groups. Figure 2 shows that, for control infants, $\log$ (SDDRR) increased at $15 \mathrm{~s}$ pre-tilt (i.e. at the first time tilting effects could have been manifested in SDDRR) and peaked at $10 \mathrm{~s}$ before tilting. Moreover, $\log$ (SDDRR) remained elevated until $10 \mathrm{~s}$ after tilting but then dropped abruptly to levels that were maintained through the post-tilt segment. The responses of PCE infants were comparatively slow. At $15 \mathrm{~s}$ before tilt, there was no increase in $\log$ (SDDRR). Then $\log$ (SDDRR) increased steadily, beginning at $10 \mathrm{~s}$ before tilt and continuing to a peak at $10 \mathrm{~s}$ after tilt. Notably, $\log$ (SDDRR) did not return to normal levels in the post-tilt segment.

$\log (\operatorname{corSDRR})$. The data are summarized in Fig. 3. Neither main effect for group $(p=0.373)$ nor interaction between group and time $(p=0.372)$ was significant. Even so, the

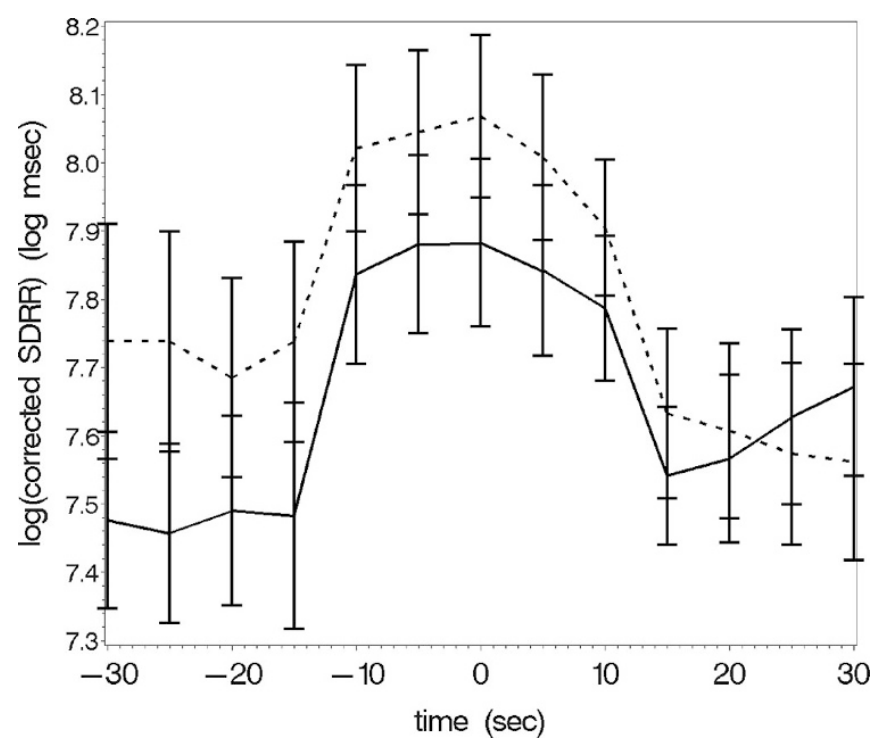

Figure 3. Group means and standard errors for log (corrected SDRR) within 30 s of tilting, - Cocaine $(n=21)$; --- Control $(n=23)$.

highly significant main effect for time $(p<0.001)$ warrants examining the trajectories of $\log$ (corSDRR) for all babies; log (corSDRR) increased significantly during the intra-tilt segment from pre-tilt (increase $=0.24 \pm 0.04 \log \mathrm{ms}, p<$ 0.0001 ), with subsequent significant decrease at post-tilt segment (decrease $=0.23 \pm 0.04 \log \mathrm{ms}, p<0.001$ ). Similar trajectories for log (corSDRR) for the PCE infants and controls were observed with $\log$ (SDRR) measurements with orthostatic stress.

Wavelet analysis. For concordance with time-domain analyses, LF wavelet power was defined as the integral of the LF wavelet power function over either the $15 \mathrm{~s}$ before tilting (horizontal position) or the $15 \mathrm{~s}$ after tilting (head-up position). There was a significant main effect for group ( $p=$ 0.039), attributable to the lower LF wavelet power for PCE infants in both the horizontal $(29.43 \pm 4.56$ versus $40.54 \pm$ 5.77 for control) and head-up positions (28.24 \pm 5.24 versus $41.26 \pm 7.66$ for control). However, there was neither a significant main effect for position nor a significant interaction between group and position. The analogous LMM for HF wavelet power yielded no significant results.

Power spectrum analysis. The stationarity assumption required for the PSA would not be tenable if we confined attention to the 15-s intervals immediately before and after tilting. Hence, the PSA entailed comparisons of $\mathrm{P}_{\mathrm{L}} / \mathrm{P}_{\mathrm{T}}, \mathrm{P}_{\mathrm{H}} / \mathrm{P}_{\mathrm{T}}$, and $\mathrm{P}_{\mathrm{L}} / \mathrm{P}_{\mathrm{H}}$ based on the 5-min intervals immediately before and after tilting. For controls, there was a significant decrease in $\mathrm{P}_{\mathrm{L}} / \mathrm{P}_{\mathrm{T}}$ from $0.80 \pm$ to $0.75 \pm 0.03(p=0.023)$ after tilt and a significant increase in $\mathrm{P}_{\mathrm{H}} / \mathrm{P}_{\mathrm{T}}, 0.20 \pm 0.02$ to $0.25 \pm 0.0 .03$ $(p=0.023)$. For PCE infants, there were no significant changes in any of the ratios. There were no significant differences between PCE and control infants in any of the ratios either pre- or post-tilt.

\section{DISCUSSION}

This study has elucidated the effects of PCE on ANS regulation of cardiovascular function, documenting a previ- 
ously unreported interaction between PCE and orthostatic stress. The interaction was most perceptible in the timedomain analyses of HR and log (SDDRR). While the controls had instantaneous but transient responses to the orthostatic stress, PCE infants had responses that were both delayed and prolonged. Although the frequency-domain analyses also made some distinction between controls and PCE infants, the inherent condensation of information associated with power spectral analyses may have limited what could be inferred from them.

Tachycardia in PCE infants may be attributed to increased sympathetic nervous system activity after exposure to a sympathomimetic drug. The increased sympathetic activity following orthostatic stress may represent an exaggerated carotid baroreceptor response. Head tilt-up position results in a small transient decrease in BP initiating a neurally mediated compensatory vasoconstriction and increase in heart rate (23). While we did not measure BP in our infants, Andreissen (24) and others have described maturation of baroreceptor reflex sensitivity (BRS) as infants reach term gestation. Thus in our control infants we can assume mature BRS and their HR changes reflect their BRS and autonomic cardiovascular control. While tilting led to elevations in HR for both the PCE and control infants in this study, the differences between groups suggest alterations in the neural control mechanisms in infants with PCE based on differences as to time to respond, degree of increase in HR, and duration of response.

HRV has been found increased, decreased, or essentially unchanged $(10,12,13,25)$, for cocaine-exposed infants vis-avis controls. Our study, partly motivated by the presence of such conflicting results in the literature, found an interaction between PCE and orthostatic stress. This interaction was most evident with $\log$ (SDDRR) as the measure of HRV. Cocaineexposed infants had decreased HRV in the pre-tilt and intratilt segments compared with controls, but they had similar or increased HRV in the post-tilt segment. Moreover, the effects of cocaine exposure on ANS regulation of cardiovascular function were manifested as differences in the times to peak HRV and the duration of elevated HRV.

For both PCE and controls, orthostatic stress led to increases also in $\log$ (SDRR), and log (corrected SDRR). The similar trajectories for $\log$ (SDRR) and log (corSDRR) suggest that both groups responded to tilt with changes (increases) in HRV. These increases in the presence of elevated HR likely reflect both sympathetic and vagal modulation in both PCE and controls. However the lower HRV measurements in exposed infants compared with controls pre- and intra-tilt, suggest the predominance of sympathetic modulation in the ANS control of cardiovascular function in PCE. In eliciting cardiovascular response to a different stress, DiPietro et al. reported shortened RR or increase in HR following an auditory stimulus, and with a trend to an increase in vagal tone (25).

The present study addressed how PCE affects responses from the sympathetic adrenal medullary (SAM) axis. In general, stressors can elicit responses from both the SAM and hypothalamic pituitary adrenal (HPA) axes. Animal studies also have demonstrated that ACTH and glucocorticoids con- tribute to cardiovascular regulation through modulation of the baroreceptor sensitivity and sympathetic activity $(26,27)$. Effects for the HPA axis are usually quantified by differences in subjects' salivary cortisol levels before and after exposure to stressors. Magnamo (28) reported that, after being subjected to both noninvasive and invasive stressors, cocaine-exposed infants had significantly lower levels of cortisol than controls and that the effects of cocaine did not dissipate with the clearance of cocaine from an infant's system. Further, the lower cortisol levels may explain the state regulatory problems noted in PCE. Levine (29) found that higher cortisol levels were associated with better scores on measures of state regulation. More investigation is required to determine the mechanisms of suppressed cortisol response attributable to PCE (28). Studies will need also to examine the integration of the responses of the two systems (SAM and HPA) to stress.

Some studies have speculated that prenatal cocaine exposure may have long-term effects on autonomic function. A prospective cohort study by Mehta et al. (11) found that infants with PCE still had altered autonomic function at 2-6 mo of age. Although studies $(30,31)$ suggest that vagal tone increases in normal children aged 3-6, it is not clear whether the same is true of children who were exposed to cocaine in utero. Further research is needed to clarify to what extent the effects of prenatal cocaine exposure persist into childhood, especially in response to stress. Investigators have also correlated the changes in HR and HRV according to levels of cocaine exposure and according to sleep states. We were not able to address these in our study and thus, this is a limitation. Also, with our small sample, it was not possible to analyze for the confounding effects of other drugs. Nevertheless, we were able to characterize the effects of PCE on cardiovascular control when BRS response is elicited. More importantly, we observed that the effects of PCE on the neonatal ANS could be demonstrated through the instantaneous HR and HRV changes in response to a stimulus such as the orthostatic stress.

\section{REFERENCES}

1. Sahni R, Schulze KF, Kashyap S, Ohira-Kist K, Fifer WP, Myers MM 2000 Maturational changes in heart rate and heart rate variability in low birth weight infants. Dev Psychobiol 37:73-81

2. Schechtman VL, Raetz SL, Harper RK, Garfinkel A, Wilson AJ, Southall DP, Harper RM 1992 Dynamic analysis of cardiac R-R intervals in normal infants and in infants who subsequently succumbed to the sudden infant death syndrome. Pediatr Res 31:606-612

3. Hermida RC, Ayala DE, Fernandez JR, Fraga JM 1994 Hardware-software approach for neonatal cardiovascular risk estimation. Biomed Instrum Technol 28:43-51

4. Karin J, Hirsch M, Akselrod S 1993 An estimate of fetal autonomic state by spectral analysis of fetal heart rate fluctuations. Pediatr Res 34:134-138

5. Sibony O, Fouillot JP, Benaoudia M, Benhalla A, Oury JF, Sureau C, Blot P 1994 Quantification of the fetal heart rate variability by spectral analysis of fetal wellbeing and fetal distress. Eur J Obstet Gynecol Reprod Biol 54:103-108

6. Hadeed AJ, Siegel SR 1989 Maternal cocaine use during pregnancy: effect on the newborn infant. Pediatrics 84:205-210

7. Bauer CR, Shankaran S, Bada HS, Lester B, Wright LL, Krause-Steinrauf H, Smeriglio VL, Finnegan LP, Maza PL, Verter J 2002 The Maternal Lifestyle Study: drug exposure during pregnancy and short-term maternal outcomes. Am J Obstet Gynecol 186:487-495

8. Bauer CR, Langer JC, Shankaran S, Bada HS, Lester B, Wright LL, KrauseSteinrauf H, Smeriglio VL, Finnegan LP, Maza PL, Verter J 2005 Acute neonatal effects of cocaine exposure during pregnancy. Arch Pediatr Adolesc Med 159:824 834

9. Oriol NE, Bennett FM, Rigney DR, Goldberger AL 1993 Cocaine effects on neonatal heart rate dynamics: preliminary findings and methodological problems. Yale J Biol Med 66:75-84 
10. Mehta SK, Super DM, Salvator A, Singer L, Connuck D, Fradley LG, Harcar-Sevcik RA, Kaufman ES 2001 Heart rate variability in cocaine-exposed newborn infants. Am Heart J 142:828-832

11. Mehta SK, Super DM, Connuck D, Kirchner HL, Salvator A, Singer L, Fradley LG, Kaufman ES 2002 Autonomic alterations in cocaine-exposed infants. Am Heart J 144:1109-1115

12. Regalado MG, Schechtman VL, Del Angel AP, Bean XD 1996 Cardiac and respiratory patterns during sleep in cocaine-exposed neonates. Early Hum Dev 44:187-200

13. Garde S, Regalado MG, Schechtman VL, Khoo MC 2001 Nonlinear dynamics of heart rate variability in cocaine-exposed neonates during sleep. Am J Physiol Heart Circ Physiol 280:H2920-H2928

14. Regalado MG, Schechtman VL, Khoo MC, Shin J, Bean XD 1998 Sources of heart rate variation during sleep in cocaine-exposed neonates. Ann N Y Acad Sci 846:415-418

15. Mansier P, Clairambault J, Charlotte N, Medigue C, Vermeiren C, LePape G, Carre F, Gounaropoulou A, Swynghedauw B 1996 Linear and non-linear analyses of heart rate variability: a minireview. Cardiovasc Res 31:371-379

16. Coumel P, Hermida JS, Wennerblom B, Leenhardt A, Maison-Blanche P, Cauchemez B 1991 Heart rate variability in left ventricular hypertrophy and heart failure, and the effects of beta-blockade. A non-spectral analysis of heart rate variability in the frequency domain and in the time domain. Eur Heart J 12:412-422

17. Neuman MR, Watson H, Mendenhall RS, Zoldak JT, Di Fiore JM, Peucker M, Baird TM, Crowell DH, Hoppenbrouwers TT, Hufford D, Hunt CE, Corwin MJ, Tinsley LR, Weese-Mayer DE, Sackner MA 2001 Cardiopulmonary monitoring at home: the CHIME monitor. Physiol Meas 22:267-286

18. McDonald R, Jenkins J, Arzbaecher R, Thorne R 1989 A software trigger for intracardiac waveform detection with automatic threshold adjustment. IEEE Computers in Cardiology Proceedings, IEEE Computer Society Press, Washington, DC, pp $167-170$

19. Regalado MG, Schechtman VL, Khoo MC, Bean XD 2001 Spectral analysis of heart rate variability and respiration during sleep in cocaine-exposed neonates. Clin Physiol 21:428-436
20. Van Ravenswaaij-Arts C, Hopman J, Kollee L, Stoelinga G, Van Geijn H 1994 Spectral analysis of heart rate variability in spontaneously breathing very preterm infants. Acta Paediatr 83:473-480

21. Dykes FD, Ahmann PA, Baldzer K, Carrigan TA, Kitney R, Giddens DP 1986 Breath amplitude modulation of heart rate variability in normal full term neonates. Pediatr Res 20:301-308

22. Jimenez RF, Gunther B, Salazar A 1997 Continuous wavelet transform of aortic pressure oscillations in anesthetized dogs: effects of 45 degrees tilting. Biol Res 30:53-64

23. Borst C, Wieling W, van Brederode JF, Hond A, de Rijk LG, Dunning AJ 1982 Mechanisms of initial heart rate response to postural change. Am J Physio 243:H676-H681

24. Andriessen P, Oetomo SB, Peters C, Vermeulen B, Wijn PF, Blanco CE 2005 Baroreceptor reflex sensitivity in human neonates: the effect of postmenstrual age. J Physiol 568:333-341

25. DiPietro JA, Suess PE, Wheeler JS, Smouse PH, Newlin DB 1995 Reactivity and regulation in cocaine-exposed neonates. Infant Behav Dev 18:407-414

26. O'Connor SJ, Gardner DS, Ousey JC, Holdstock N, Rossdale P, Edwards CM, Fowden AL, Giussani DA 2005 Development of baroreflex and endocrine responses to hypotensive stress in newborn foals and lambs. Pflugers Arch 450:298-306

27. Segar JL, Van Natta T, Smith OJ 2002 Effects of fetal ovine adrenalectomy on sympathetic and baroreflex responses at birth. Am J Physiol Regul Integr Comp Physiol 283:R460-R467

28. Magnano CL, Gardner JM, Karmel BZ 1992 Differences in salivary cortisol levels in cocaine-exposed and noncocaine-exposed NICU infants. Dev Psychobiol 25:93103

29. Levine S 1962 Plasma-free corticosteroid response to electric shock in rats stimulated in infancy. Science 135:795-796

30. Goto M, Nagashima M, Baba R, Nagano Y, Yokota M, Nishibata K, Tsuji A 1997 Analysis of heart rate variability demonstrates effects of development on vagal modulation of heart rate in healthy children. J Pediatr 130:725-729

31. Finley JP, Nugent ST 1995 Heart rate variability in infants, children and young adults. J Auton Nerv Syst 51:103-108 